\title{
CARDIAC SYNDROMES COMPLICATING DIABETES AND THEIR TREATMENT
}

\author{
BY
}

\author{
K. SHIRLEY SMITH
}

Received August 4, 1942

While it is true that common diseases must often co-exist in the same patient, the frequent association of heart disease with diabetes is the expression of a causal relationship. In the present paper the evidence for this statement will be examined. Thereafter an analysis will be made of 49 patients with heart disease and diabetes, with a discussion of the nature of the anginal and other cardiac syndromes developing, and typical clinical examples will be mentioned. Lastly, schemes of treatment for the various combinations of these maladies will be outlined.

That diabetes conduces to early, serious, and often fatal cardiovascular disease has been suspected for more than fifty years (Vergely, 1881; Frerichs, 1883), but pathological and clinical data have become available chiefly during the past decade. Blotner (1930) in necropsies of 77 diabetics found conspicuous disease of the coronary arteries in 45 per cent ; cardiac infarction was the cause of death in 10 per cent, the ages ranging from 13 to 85 years; these figures contrasted with a 21 per cent incidence of coronary sclerosis in 450 non-diabetics between the ages of 40 and 80 years. Warren (1930) found coronary thrombosis and cardiac infarction in 12 per cent of necropsies among diabetics, and commented on the frequency with which diabetics with advanced coronary sclerosis escaped angina. Root and Graybiel (1931), in a study of 210 cases of diabetes with angina, stated their experience that at necropsy every patient with a five-year history of diabetes had some degree of coronary sclerosis: they drew their 210 anginal patients from a series of 7000 diabetics, an incidence far in excess of that in an ordinary hospital population. Moreover, they found that diabetes exerted a levelling influence upon the sex incidence of angina (122 males and 88 females). For every patient in whom angina preceded diabetes, there were 21 in whom diabetes had preceded angina.

A comparison of the atherosclerotic lesions found at necropsy among 349 diabetics and those found in 3400 non-diabetics has been made by Root, Bland, Gordon, and White (1939). Coronary occlusion was much more frequent among the diabetics ( 23 compared with 6 per cent, between the ages of 40 and 60 ). In the same age group, 17 per cent of diabetics had coronary narrowing without occlusion, as against 12 per cent in non-diabetics. Clinical studies leading to similar conclusions have been made by Friedman (1935), who demonstrated aortic sclerosis by clinical methods in 75 per cent of 120 diabetics over 39 years of age. The high incidence of hypertension in diabetes has been shown by Root and Sharkey (1936); high blood pressure was present in 54 per cent of their series of 175 diabetics. They noted that coronary changes were absent only when diabetes was of less than three years' standing, and that advanced coronary sclerosis was twice as frequent in diabetics as in non-diabetics. Bell and Clawson (1928) found hypertension in 42 per cent of diabetics over the age of 50 . Parallel to these clinical findings were the observations of Lehnherr (1933) who compared the cholesterol and calcium contents of aortas from diabetics and non-diabetics, and found exaggerated lipoid changes and greater calcium and phosphorus deposits in the diabetic aortas.

These pathological and clinical facts relating to the heart and aorta are consistent with the wellestablished relationship between diabetes and arteriosclerosis. That diabetes conduces to early and progressive general arteriosclerosis is no longer open to question in the light of the studies of Heitz (1923), Joslin (1927), Shepardson (1930), Priscilla White (1932), Leary (1935), and Root and Sharkey (1936). Shepardson gave radiological evidence of a 36 per cent incidence of calcification in the arteries of the legs in diabetics up to the age of 40 , the average being 23 years. White gave similar results among diabetic children up to 15 years of age (19 out of 106 cases). Although these results strongly suggested that the diabetic condition conduced to arteriosclerosis, it could still be argued that arteriosclerosis and diabetes took origin from some common antecedent constitutional abnormality.

The work of Root and Sharkey (1936) gave additional evidence of a causal relation between diabetes and arteriosclerosis. They compared the necropsy findings of 175 diabetics of all ages with 
those of 170 non-diabetics all over 40 years of age. A striking excess of arteriosclerotic lesions was found in the former group, but there was still the possibility that the arterial lesions began before the diabetes. To elucidate this point they investigated the arterial condition at necropsy of 23 diabetics of less than one year's duration. This group showed rather less arterial change than a control group of 170 non-diabetics. A similar disparity was recorded in the condition of the coronary arteries in the two groups. Moreover, as Root and Graybiel (1931) have pointed out, diabetes most frequently develops at 51 years, while diabetic angina occurs most commonly in the decade in which ten-year cases of diabetes are most numerous. These considerations, coupled with the levelling influence of diabetes upon the sex distribution of angina, point unmistakably to diabetes with its associated metabolic change as contributing to bring about arteriosclerosis in general and decrease of the coronary artery in particular. As Levine (1929) has demonstrated, diabetes must be accorded a place only subsidiary to that of high blood pressure among the morbid states conducing to coronary thrombosis.

\section{Clinical Observations in Present Series}

The present observations are based upon 49 consecutive patients in whom diabetes co-existed with cardiovascular disease. The diabetes was of every grade from symptomless mild diabetes, proved by sugar tolerance curves, to gravely toxic or comatose stages. Cardiovascular disease similarly ranged from symptomless hypertension to acute coronary occlusion with high grades of shock. Diabetes accompanied only by peripheral arterial disease was not included. The sex distribution was fairly even: 26 females to 23 males. The average age of the women was 61 , that of the men 62 years. The cardiovascular disease was always founded on hypertensive or degenerative lesions or both ; no example of rheumatic, syphilitic, or thyrotoxic heart disease in association with diabetes was encountered. But although the ætiology of the cardiac complications was strictly limited, a wide range of disorders of function was observed, such as left ventricular failure with cardiac asthma, congestive failure with œdema, and various forms of angina.

A very frequent accompaniment of diabetes was hypertension; this was present in 41 cases and in 3 others there was evidence that it had previously existed, making 44 out of the total of 49 cases. In general the grade of hypertension was far from trivial; in 31 instances the systolic blood pressure was 180 or more, compared with 13 in which it was under $180 \mathrm{~mm}$. As striking as the prevalence of hypertension was the reduction in blood pressure during treatment. This fact and the observation that the reduction of pressure during stabilization of the diabetes was not uncommon, even in patients previously confined to bed, suggested that hypertension in these cases was in part attributable to the immediate metabolic fault.

\section{Relation of Cardiovascular Disease to Diabetes}

The patients comprising the present series fell naturally into certain categories. Some were aware of the existence of heart disease and diabetes at the time they first came under observation; of these a few had been or were still on insulin treatment, while others had never received this. Frequently a patient known to be diabetic presented himself with the initial symptoms of cardiovascular disease; rarely the converse relationship held. A well-defined group were those who had regarded themselves as well but now manifested symptoms of diabetes or of heart disease and were subsequently shown to possess a cardiopathy or diabetes respectively.

\section{Group I. Patients known to have diabetes and cardiovascular disease (18 of present series).}

These are often diabetics of long standing and the disease may be of a grade to require insulin treatment. It is well known that too great a dosage of insulin may provoke an anginal attack and this may culminate in coronary thrombosis. Equally, congestive heart failure or left ventricular failure may develop by the same mechanism. On the other hand, the dosage may be too small (actually, or relatively as a result of intercurrent infection). Coronary thrombosis is specially apt to occur in the diabetic, but there is evidence that inappropriate dosage of insulin may conduce to this accident. Some patients may never have had insulin treatment, although obviously in need of it when they come under observation. This commonly arises through the diabetes being at first slight and easily controlled by diet alone; gradually the fault becomes more severe and perhaps the patient has ignored warning sysmptoms of this process; finally, a cardiac attack discloses the untrolled or untreated diabetes. Evidence that the onset of effort angina, coronary thrombosis, cardiac asthma, or chronic congestive failure is attributable to the diabetic fault will be adduced below. 
Group II. Patients known to have diabetes, developing cardiovascular symptoms (12 cases).

Patients of this group may seek advice on account of slight disorders, such as undue fatigue, shortness of breath, bouts of dizziness, or attacks of faintness. In a few cases one of the major seizures mentioned in the preceding section may be the first indication that cardiovascular disease has been developing.

Group III. Patients known to have cardiovascular disease, developing symptoms due to diabetes (5 cases).

The fact that relatively so few patients belong to this category is consistent with the established fact that diabetes is important in the ætiology of heart disease. In view of this relationship it would be surprising if many patients experienced symptoms of cardiac lesions before those of diabetes.

Group IV. Patients previously believed well, in whom the occurrence of a cardiac or a diabetic syndrome brings to light the existence of diabetes or heart disease respectively (14 cases).

It is remarkable that in so many patients diabetes and its cardiac complication may reach a fairly advanced stage without either of them causing obtrusive symptoms, but it is in keeping with the observation of Warren (1930) and other workers that necropsy in the diabetic may disclose advanced coronary sclerosis although there had been no symptoms of angina. In general it is uncommon for a grave diabetic development to occur in heart disease, or for a serious cardiac attack to complicate diabetes unless the patient has had early symptoms of one or other or both maladies previously.

While it might be more logical to consider the various cardiac disorders that may be observed in these groups of patients, it will save repetition if cardiac syndromes are reviewed in turn, the various circumstances in which each may occur being shown by reference to illustrative cases. The following will be dealt with : effort angina, coronary thrombosis, cardiac asthma, and congestive heart failure.

\section{VARIOUS CARDIAC SyNDRomes}

Effort angina and spasmodic angina. This may be the natural and not unexpected expression of coronary sclerosis, and may develop in the manner so often seen in patients who have no diabetes. Nevertheless, while it is obvious that the heart of the diabetic must be provided with more than sufficient sugar for its needs, the failure to utilize this source of energy may lead to anginal attacks that are abolished by the use of insulin. Hetenyi (1927) has reported examples. This process has been observed in the present study in a man aged 53 in whom bouts of pain and oppression in the chest became much less frequent and severe as the fasting blood sugar fell from 315 to $219 \mathrm{mg}$. per 100 c.c. on treatment by insulin, 10 units twice daily, and a diet of 1800 calories. (Case I.)*

The rapid fluctuations of blood sugar that may take place from various causes in the diabetic patient who is on insulin treatment entail sudden alterations in the nutrition of the heart. As effort angina may correspond to these phases of imbalance, this indicates that anginal pain in the diabetic (and perhaps also in the non-diabetic) is not only related to ischæmia but also to metabolic disorders in the heart muscle, a phenomenon discussed in a previous publication (Shirley Smith, 1933). Angina produced by an excessive lowering of blood sugar in a patient taking too large a dose of insulin has been called " insulin angina". Fortunately no syndrome of this kind was evoked during treatment in any cases of the present series.

Coronary thrombosis and cardiac infarction. Coronary thrombosis occurs with greater frequency among diabetics than among non-diabetics of the same age group. Its incidence is greater among diabetics who need, but are not taking, insulin. Thus an elderly female with long-standing glycosuria and hypertension developed increasing fatigue as a result of undue activity. After transient anginal attacks on three successive nights she developed coronary thrombosis, with congestive heart failure. By the use of digitalis and insulin therapy and the restriction of her fluid intake to 2 pints in 24 hours, she recovered and remained in good health for over three years when heart failure recurred. (Case 2.)

Patients who are on insulin treatment for diabetes are by no means protected from coronary thrombosis, even though the correct dosage seems to have been found. In a woman who was admitted with a ten-year history of diabetes, lethargic, dehydrated, and stuporous, the immediate danger was averted, but in spite of apparently successful adjustment of insulin dosage, signs of collapse recurred. Death ensued 19 hours after the onset, and necropsy confirmed the diagnosis of coronary thrombosis. (Case 3.)

When patients are on treatment by diet and insulin the sudden reduction or withdrawal of insulin may mark the onset of coronary thrombosis. In one instance the development of the attack followed

* Full case histories were submitted, but unfortunately had to be left out owing to reduction in the paper allowance.-EDITOR. 
the omission of a dose of insulin the previous evening. A blood sugar level of $600 \mathrm{mg}$. per 100 c.c. showed that there had been progressive loss of control of the diabetes through inadequate dosage of insulin. (Case 4.)

It is not unusual for coronary thrombosis to occur as the initial expression of heart disease in a patient known to be diabetic. In some instances it develops with increasing hyperglycæmia and acetonæmia: in others diabetic coma may be the immediate sequel of acute coronary occlusion ; e.g. a woman diabetic, aged 55, had an attack of coronary thrombosis, and about 60 hours after the onset she had become unconscious and extremely restless, and the blood sugar was found to be $650 \mathrm{mg}$. per 100 c.c.; she died very soon after the institution of treatment. (Case 5.)

While it is common for heart disease to develop in the diabetic the reversal of the order is comparatively rare. Only 5 examples were observed in the present series of 49 patients. In one, a female of 63, there was a history of effort angina for three years; during an acute pulmonary infection glycosuria was observed for the first time. (Case 6.) In another, a physician aged 57, there was undue fatigue six months after recovery from coronary thrombosis; he was found to have a low renal threshold, but the fact that his condition conspicuously improved with reduction of carbohydrate intake suggested that a fault of metabolism also existed. (Case 7.)

Coronary thrombosis often occurs without any warning symptoms of heart disease, and routine examination at the time may bring to light the existence of diabetes. This sequence of events was recorded in a female, aged 54, who complained essentially of pain in the left arm. Routine examination disclosed diabetes; she was admitted to hospital with evidence of a recent TIII type of cardiac infarc tion. She recovered and was discharged on a weighed diet of 2000 calories and 10 units of insulin daily, which allowed intermittent glycosuria. Death occurred suddenly a few months later. (Case 8.)

The intensity of arterial disease and its rapid progress in the diabetic offer an obvious explanation of the frequency with which anginal syndromes are observed. When cardiac breakdown occurs in forms that represent myocardial as distinct from coronary failure, the relationship is not so clear. In some patients in the present series, cardiac asthma was a circulatory expression of uncontrolled diabetes, suggesting that left ventricular failure might result from an untreated or a badly corrected fault in carbohydrate metabolism. Syndromes of this order suggest that diabetes exerts a deleterious effect on the myocardium, not only by virtue of coronary sclerosis but also through direct impairment of the parenchyma of the heart. That general heart failure with congestion and odema may be the consequence of untreated diabetes will be suggested later, but the fact may be referred to at this point as demonstrating further the myocardial (as distinct from coronary) damage that may develop.

Cardiac Asthma. Cardiac asthma, following the aggravation of a latent diabetes associated with long-standing hypertension, was observed in the following instance. A woman, aged 41, had severe hypertension, and was seen at intervals for five years, glycosuria being found on one occasion. Twenty-four hours after a motor accident in which she was shocked but not injured, she experienced thirst and on the following night had cardiac asthma. Investigation disclosed severe diabetes. At the time of discharge on a fixed diet of 2000 calories, with insulin, 7 units twice daily, she was free from cardiac or diabetic symptoms, but showed intermittent glycosuria. (Case 9.)

It is not surprising that left ventricular failure and ultimately general congestive failure sometimes succeed effort angina in diabetes, as this sequence of events is commonly seen in coronary sclerosis without diabetes. In Case 10 cardiac asthma was the presenting symptom at the time of admission. A male, aged 58, had been a diabetic taking insulin for 7 years. For 18 months he had experienced effort angina. Cardiac asthma had supervened, and later signs of congestive heart failure. After admission to hospital and adjustment of insulin dosage, the paroxysms of dyspnœa came to an end and the signs of failure vanished. These events might be explicable on the basis of rest alone, were it not for the fact that rest had been employed without success prior to admission and that in hospital no relief from the cardiac asthma was obtained until adjustment of insulin dosage had been effected.

In another instance a woman, aged 60, had suffered for several years with diabetes and hypertension. Paroxysmal dyspnœa, chiefly nocturnal, developed, and relief from these distressing attacks synchronized with adjustment of the insulin dosage and diet. (Case 11.)

Congestive heart failure. While coronary arterial disease finds its most characteristic expression in anginal attacks of various kinds, it may culminate in congestive heart failure without painful episodes at any time. The development of failure in the diabetic would therefore not be surprising, and it might be regarded as entirely independent of the diabetic state except in so far as the latter conduced to coronary sclerosis. The disturbances of cardiac nutrition in diabetes may play a part in the development of failure, and stabilization may bring improvement in the circulatory condition. A man of 69 was admitted with congestive heart failure; he had been diabetic for 33 years. Severe dyspnœa persisted in spite of control of the hyperglycæmia and acetonæmia. Blood sugar examination showed that too great a reduction had been secured $(56 \mathrm{mg}$. per 100 c.c.). A reduction in insulin 
dosage allowed the blood sugar to rise to $222 \mathrm{mg}$. and signs of failure progressively disappeared. Failure re-appeared a month or two later, and on several occasions during this phase intravenous injections of glucose relieved severe paroxysms of dyspnœa. (Case 12.)

In another case of congestive failure following cardiac infarction, a choice had to be made between a free fluid intake to combat impending coma, and a restricted intake indicated by the orthopnoa and congestive failure. Further reference to the therapeutic dilemma is made hereafter in the consideration of treatment.

\section{Treatment of the Cardiac Diabetic}

As has been shown, the syndromes resulting from the cardiac complications of diabetes are of great variety. They are in fact often the same as those met with in patients who are not diabetic; in these treatment will be primarily directed to the circulatory condition, but treatment of the metabolic fault may never be ignored with impunity. In others the clinical features represent a blend between the effects of the cardiovascular disease and nutritional and toxic consequences of the diabetes; here treatment will call for an appraisal of the relative importance of the two elements: since the requirements of the one may be at variance with those of the other, great difficulty may be experienced in deciding upon the most suitable scheme of treatment. In yet others circulatory collapse of the second type described by Frerichs (1883) may complicate diabetic coma without any indication of congestive failure or coronary thrombosis. Experience shows that many of such patients may in the initial grave cardiac attack succumb within a few hours or days, the falling blood pressure and progressive collapse resisting all therapeutic efforts. The treatment of syndromes of this type has been studied by many workers (Labbe \& Boulin, 1928 \& 1933; Lawrence, 1933; and Clerc, 1934). Equally, the patient may be treated with the necessary precautions to be mentioned below, may make great improvement in the heart and diabetes, and yet may die suddenly without obvious immediate cause when he appeared to be well on the way to convalescence. These difficulties have been described by several observers (Haynal, 1925; and Soskin, Katz, Strouse, \& Rubinfeld, 1933).

When a cardiac disease such as effort angina supervenes in a diabetic whose condition has been long recognized and kept under control, treatment will naturally first be directed to the necessary adjustments in the mode of life and to appropriate medicinal measures for the relief and prevention of pain. Nevertheless the development of angina will prompt an immediate survey of the diabetic state. While it has long been regarded as unwise to treat vigorously by diet diabetes in later life, it is specially dangerous to treat diabetes in the cardiac patient as a pure problem of diabetic stabilization. Sudden alterations of blood sugar by diet or insulin are not well tolerated. Treatment on the metabolic side will therefore be directed essentially to the avoidance of acetonæmia; if glycosuria persists in spite of qualitative or maintenance dieting, insulin may be used, but always in less quantity than would be employed if the same patient were free from signs of a cardiac fault. In general, it is safer for these patients to live with a moderate hyperglycæmia and to have glycosuria from time to time, than to be strictly controlled and rendered constantly free from sugar. This is because the myocardium may depend for its nutrition upon hyperglycæmia off-setting the deficient coronary flow.

Diet will be an important element in the treatment of all cardiac diabetics. Sometimes general and qualitative restriction (of bread, sugar, potatoes) is all that is required, but in many cases a full quantitative system will be necessary, with the number of calories laid down. The construction of such a diet with sufficient variety will not be difficult on the Lawrence line-ration scheme. Cardiac diabetics and in fact all diabetics should restrict foods of high cholesterol value such as eggs, cream, butter, cheese, and brains.

More complex situations arise when the diabetic develops such a condition as congestive heart failure or left ventricular failure with cardiac asthma. Here again the cardiovascular syndrome may be no different from one that might be seen apart from diabetes, and may seem to bear little relation to the metabolic fault, but as we have endeavoured to show in the cases mentioned, the therapeutic test may indicate that a close relationship exists between the circulatory breakdown and some maladjustment or failure of management of the diabetes. If no insulin was previously needed it may become necessary, owing to the increased basal metabolism in heart failure, to fever which sometimes accompanies failure, or to the disorganization of digestion and assimilation caused by congestion. On the other hand, if insulin were being taken the dose might need to be increased or decreased. Aggravation may be due equally to excess or deficiency of insulin.

Estimations of blood sugar (both fasting and three-quarters of an hour after a meal) coupled with frequent examination of the urine for sugar and acetone bodies will be essential. The ferric chloride (Gerhardt) test will in the first instance give more assistance than the Rothera test by providing a demarcation between grosser and milder grades of ketosis. If the ferric chloride test and clinical features show that there is little or no ketosis, the immediate danger of the patient is little more 
than might be attributed to the heart failure alone. A careful review of the metabolic condition and analyses of blood and urine will be essential, and it may or may not be found necessary to give insulin or to adjust a dosage previously in use. In any case rather less insulin will be given than would be the case if no cardiac lesion were manifest. If the ferric chloride test is positive, and particularly if other indications of severe ketosis are present (rising pulse, abdominal pain, furred tongue, and headache), treatment of the diabetes will become of preponderating importance and urgency. The intake of fluid by mouth will be increased to six or eight ounces each hour, and insulin and glucose in the proportion of one unit of insulin to rather more than one gram of glucose. As the occasion will be one of great urgency, no accurate weighing of glucose will be practicable; roughly, a full (not heaped) tablespoonful of glucose contains fifteen to sixteen grams. Therefore a dose of twentyfive or thirty units of insulin would be suitably combined with two tablespoonfuls of glucose; these doses might be repeated once or more at intervals of two to four hours according to the needs of the individual case. Insulin will usually be given intramuscularly, but may be given intravenously if the condition is grave; the glucose will be given orally whenever possible, but if not, by rectal or intravenous infusion. It is obvious that each case will require assessment on its merits and that no hard and fast rules can be laid down. Nevertheless, the following points are of great importance in all cases in which a cardiovascular lesion of more than trivial grade is accompanied by diabetes with ketosis or impending coma.

1. The situation is grave and therapy urgent.

2. No time should be lost awaiting the results of laboratory investigations.

3. Immediate attention should be paid to the essentials of treatment for the diabetic complication, by the provision of warmth and rest, the increase of fluids by mouth, and the provision of stimulants, such as hot coffee or tea.

4. The use of insulin, coupled with more than balancing amounts of glucose added to the drinks or the infusions, is important.

5. From the cardiac standpoint warmth and rest are equally essential, the degree of recumbency depending upon the patient's wish.

6. Repeated examinations of urine will be necessary; it should not become constantly sugar-free but the aim is to abolish ketosis; blood sugar (fasting) should be estimated every few days and levels lower than 130 or 140 are to be avoided.

The association of diabetic coma with coronary thrombosis is one of the most desperate medical emergencies. The combination may take the following forms: coronary thrombosis may occur in a previously stabilized diabetic and induce coma; a diabetic may become unstabilized through some lapse in treatment or diet, or through some intercurrent infection, and then develop coronary thrombosis. In other cases the patient passes insidiously into diabetic coma with associated symptoms suggesting coronary disorder (pain or oppression in the chest); in these it may only be the course of events that will show whether or not a coronary occlusion has occurred. If it has, there will be either a fatal issue or a very protracted and dangerous illness; if it has not, the treatment directed to the coma will usually be followed by very rapid recovery. Neither the electrocardiogram nor its evolution in serial records is of conclusive help in this diagnosis, because in coronary thrombosis no change may be seen in the first few days (which are important ones from the standpoint of treatment), while in severe diabetes without coronary complications cardiographic changes very similar to those of coronary occlusion are sometimes found (Shirley Smith \& Hickling, 1933; and Bellet \& Dyer, 1937); however, the evolution in serial records is rather different in this case.

The problem of fluid administration is often difficult and sometimes insoluble in the treatment of heart failure associated with diabetes, of coronary thrombosis complicated by diabetes, and of diabetic coma or impending coma when severe heart disease exists. It is accepted that in congestive heart failure the fluid intake should be limited to $25 \mathrm{or} 30 \mathrm{oz}$., and the same rule applies to treatment in the early days of acute coronary occlusion which has produced more than trivial constitutional upset. At the same time in each of the three examples cited, the appropriate treatment of the diabetes demands administration of perhaps four or five pints of fluid in the twenty-four hours, or if dehydration has developed, double these quantities, even if part has to be given by rectal or intravenous routes. The impossibility of reconciling these diametrically opposed requirements in the same patient is the reason why treatment is so often unavailing and why combined maladies of these kinds are so lethal.

In spite of these obvious difficulties there are certain general principles underlying fluid administration which should be observed in constructing a scheme of treatment.

In congestive heart failure accompanied by severe diabetes it is better to risk the development or increase of œdema than to allow progressive hyperglycæmia and ketosis. It will therefore be better to give sufficient fluid (in addition to other medication such as insulin) to minimize the risk of coma. Consequent œdema may be later dispersed by the mercurial diuretics, whereas increasing ketosis will 
certainly aggravate failure even though no œdema exists. If diabetic coma is present the intravenous route must be used for the administration of fluid and glucose. The signs of dehydration denote a total fluid lack of perhaps 10 to 12 pints, and large quantities of fluid must therefore be given in a relatively short time (Lawrence, 1930). In Case 4 of the present series there was no doubt that the intravenous infusion of 10 per cent glucose saline helped in the relief of dangerous collapse due to the combined effects of severe diabetic ketosis (blood sugar $600 \mathrm{mg}$. per 100 c.c.) and coronary thrombosis.

\section{SUMMARY}

The evidence proving that diabetes conduces to early and severe arteriosclerosis and, specially, to coronary arterial disease and coronary thrombosis has been reviewed.

The clinical features of 49 consecutive cases of diabetes associated with heart disease have been analysed. The sexes were fairly evenly represented; the average age of the men was 62 years, and that of the women 61 years.

Hypertension was present in 44 of the 49 patients (almost 90 per cent); in 31 instances the blood pressure was $180 \mathrm{~mm}$. or more. As is usual in hypertension uncomplicated by diabetes, the blood pressure frequently dropped swiftly and considerably on confining the patients to bed. Reasons have been given for believing that hypertension in the diabetic was in some degree directly attributable to the metabolic fault.

A study was made of the manner in which angina of effort, spasmodic angina, coronary thrombosis, left ventricular failure, and congestive heart failure supervened in diabetics. Some case histories illustrating these events and showing the relationship between the diabetic state and the cardiac development have been summarized.

As a rule, patients with diabetes and one or other form of angina or heart failure were found to derive benefit from any necessary readjustments of regime or insulin dosage. Controlled observations were not, for obvious reasons, generally possible, but in a few patients, in whom this adjustment only was made, deterioration in the heart condition was replaced by improvement.

It has been concluded that while the lives of patients with disease of a coronary artery are at serious risk those of the "cardiac diabetics" are even more precarious. Coronary thrombosis in the diabetic is extremely hazardous.

Methods of treating cardiac complications in diabetes have been reviewed. The requirements of diet, fluid, insulin, glucose, and remedies directed to the heart have been considered. The wellknown principle, that in patients with heart disease sudden reductions of blood sugar by diet or insulin are not well tolerated, has been endorsed in the present study. In general the use of insulin will be in such dosage as may be necessary to abolish acetonæmia and reduce severe hyperglycæmia. To use insulin to procure stabilization or exact control of the diabetes in patients with additional heart disease is to court disaster.

\section{REFERENCES}

Bell, E. T., and Clawson, B. J. (1928). Arch. Pathol. and Lab. Med., 5, 939.

Bellet, S., and Dyer, W. W. (1937). Amer. Heart J., 13, 72.

Blotner, H. (1930). New Engl. J. Med., 203, 709.

Clerc, A. (1934). Problèmes Actuels de Pathologie Médicale: Troubles Cardiaques du Diabète Sucré, Paris. Frerichs, F. T. (1883). Z. klin. Med., 6, 3.

Friedman, G. (1935). Arch. intern. Med., 55, 371.

Haynal, E. von (1925). Klin. Wschr., Jahrg., 4, 403.

Heitz, J. (1923). La Médicine, 4, 783.

Hetenyi, G. (1927). Wien. Arch. inn. Med., 13, 95.

Joslin, E. P. (1927). Ann. clin. Med., 5, 1061.

Labbé, M., and Boulin, R. (1928). Presse med., 36 (i), 257.

- (1933). Ibid. 41 (ii), 1705.

Lawrence, R. D. (1930). Brit. med. J., 1, 690.

Leary, T. (1935). J. Amer. med. Ass., 105, 475.

Lehnherr, E. R. (1933). New Engl. J. Med., 208, 1307.

Levine, S. A. (1929). Medicine: Coronary Thrombosis: its various Clinical Features, Vol. 8, 245).

Root, H. F., and Graybiel, A. (1931). J. Amer. med. Ass., 96, 925.

Root, H. F., Bland E. F., Gordon, W. H., White, P. D. (1939). J. Amer. med. Ass., 113, 27.

Root, H. F., and Sharkey, T. P. (1936). Ann. intern. Med., 9, 873.

Shepardson, H. C. (1930). Arch. intern. Med., 45, 674.

Smith, K. Shirley (1933). Lancet, I, 632.

Smith, K. Shirley, and Hickling, R. A. (1932). Lancet, I, 501.

Soskin, S., Katz, L. N., Strouse, S., and Rubinfeld, S. H. (1933). Arch. intern. Med., 51, 122.

Vergeley, P. (1881). Bull. Acad. Méd., 10, 1418, and Gaz. hedb. Méd. et Chir., 3, 20, 364.

Warren, S. (1930). Pathology of Diabetes, Philadelphia, p. 144.

White, Priscilla (1932). Diabetes in Childhood and Adolescence, Philadelphia, p. 178. 